\title{
Método para Estabelecimento de Referências em Ciclos de Melhoria de Processo
}

\author{
Márcia R. M. Martinez e Clenio F. Salviano \\ Centro de Tecnologia da Informação Renato Archer (CTI) \\ Rodovia D. Pedro I, km 143.6, CEP 13069-901, Campinas - SP - Brasil \\ \{marcia.martinez, clenio.salviano\} a cti.gov.br
}

\begin{abstract}
Resumo. Ciclos de melhoria de processo de software, sistemas ou serviços utilizam modelos de referência para orientar as ações e avaliar os resultados. Normalmente são considerados como modelos de referência Perfis de Capacidade de Processo (ou Niveis de Maturidade da Capacidade) tais como aqueles dos modelos do CMMI, ISO/IEC 15504 (SPICE) e MPS.BR. Porém, dois outros tipos de modelos também são utilizados, embora geralmente não sejam considerados como modelos de referência: Descrição da Realização de Processo e Indicadores de Desempenho de Processo. Este artigo apresenta um método inovador para estabelecimento integrado destes três tipos de modelos durante as fases de um ciclo de melhoria para uma melhor orientação e acompanhamento da melhoria.
\end{abstract}

Abstract. Software, system and services process improvement cycles use reference models to guide the actions and to assess the results. Usually Process Capability Profiles (or Capability Maturity Levels) such as those from CMMI, ISO/IEC 15504 (SPICE) and MPS.BR models, are considered as reference models. However, two other types of models are also used, although generally not considered as reference models: Process Enactment Description and Process Performance Indicators. This paper presents a novel method for an integrated establishment of these three types of models during process improvement phases for better guidance and monitoring of improvement.

\section{Introdução}

Este artigo apresenta um método inovador para oficinas de estabelecimento de referências para Melhoria de Processo de Software, Sistemas ou Serviços (MPS). Durante um ciclo de melhoria de processo é importante identificar e ajustar referências para orientar a melhoria. Estas referências devem estar integradas com os objetivos de negócio e com a cultura da organização. É também importante avaliar o quanto a melhoria está atingindo estas referências. Completando esta sequiência, estas referências devem ser ajustadas com base em análises das avaliações e no aprendizado. Esta sequência é chamada de Estabelecer Referências e deve ser realizada como uma oficina de trabalho na qual os atores participem. Para orientar a realização desta oficina o método descrito neste artigo está sendo desenvolvido e utilizado seguindo uma metodologia de pesquisa baseada em interações com a prática.

Este método é denominado de PRO2PI-WORK (Workshop for Establishing a PRO2PI, Oficina para Estabelecimento de PRO2PI) onde PRO2PI ( Process Modeling 


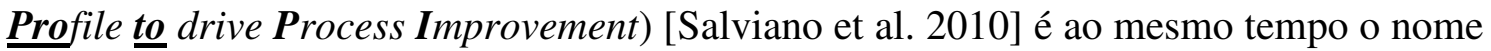
da metodologia para melhoria de processo e a denominação do conjunto de referências que orientam a melhoria. Este conjunto é formado por modelos de três tipos: Perfil de Capacidade de Processo (Process Capability Profile), Descrição da Realização de Processo (Process Enactment Description) e Indicadores de Desempenho de Processo (Process Performance Indicator). PRO2PI-WORK foi desenvolvido na metodologia PRO2PI [Salviano 2009] [Salviano et al. 2010], mas pode ser (e está sendo) utilizado também em ciclos de melhoria de processo com qualquer outra abordagem.

Este artigo está estruturado em seis seções, das quais está é a primeira. A Seção 2 apresenta a motivação do trabalho. A Seção 3 apresenta o objetivo e a metodologia utilizada. A Seção 4 descreve a versão atual do método PRO2PI-WORK. A Seção 5 descreve uma experiência de utilização do método. A Seção 6 apresenta as conclusões.

\section{Motivação}

Uma organização realiza ciclos de MPS utilizando uma abordagem, como, por exemplo, a abordagem IDEAL (Initiating, Diagnosing, Establishing, Acting, Learning) [McFeeley 1996] e o ciclo de melhoria da ISO/IEC 15504-4 [ISO/IEC 2004]. Estas abordagens incluem fases para iniciar o ciclo de melhoria, definir o Perfil de Capacidade de Processo (que pode ser um nível de maturidade) a ser atingido, avaliar as práticas correntes em relação ao perfil definido, planejar as ações de melhoria, realizar ações de melhoria e institucionalizar as melhorias. Durante estas fases tem sido importante avaliar os resultados da melhoria dos processos em relação ao perfil definido para orientar a continuação da melhoria em direção a atender o perfil definido.

Existem requisitos e métodos para avaliações de processo disponíveis, incluindo as variações A, B e C do método de avaliação de processo SCAMPI (Standard CMMI Appraisal Method for Process Improvement) [SEI 2011] em relação aos modelos do CMMI, os requisitos para processos documentados para avaliação de processo definidos pela ISO/IEC 15504 (2004) e método MA-MPS (Método de Avaliação para Melhoria do Processo de Software) definido pelo MPS.BR [SOFTEX 2009]. Estes requisitos e métodos orientam a especificação de um Perfil de Capacidade de Processo no início de uma avaliação e a realização da avaliação em relação a este perfil. Desta forma estes requisitos e métodos apóiam a melhoria de processo orientada por um perfil. Porém uma melhoria de processo não deve ser orientada apenas por um Perfil de Capacidade de Processo, mas também por pelo menos mais dois tipos de referências: Descrição de Realização de Processo e Indicadores de Desempenho de Processo.

Estes três tipos de referência para MPS já são utilizados com ênfases diferentes nos ciclos de melhoria de processo realizados atualmente. Porem apenas as referências do tipo Perfil de Capacidade de Processo são consideradas como referências para a melhoria. Uma análise apresentada em um artigo anterior (Salviano 2011) conclui que embora estes três tipos de referência sejam considerados pelos modelos mais relevantes de boas práticas utilizados para melhoria de processo, como exemplo, os modelos do CMMI (incluindo o modelo CMMI-DEV) e os modelos compatíveis com a ISO/IEC 15504 (SPICE) (incluindo o modelo ISO/IEC 15504-5 e o modelo MR-MPS do MPS.BR), existem limitações: a) os modelos do CMMI (e alguns modelos do SPICE) permitem somente o uso de um conjunto de áreas de processo pré-definido para modelos Perfil de Capacidade de Processo; b) os modelos do CMMI e SPICE 
restringem modelagem descritiva por modelos de especificação especificados anteriormente; e c) os modelos do CMMI e SPICE não exploram plenamente a integração de todos os três tipos de modelos de processos.

Existe um excesso de foco na referência do tipo Perfil de Capacidade de Processo em detrimento dos outros dois tipos de referências. Ciclos de melhoria têm sido inclusive identificados pelo modelo utilizado para o Perfil de Capacidade de Processo. Este desequilíbrio prejudica os objetivos da MPS e já foi percebido pela comunidade de MPS. Em um trabalho recente, 29 especialistas de vários países em melhoria de processo de software desenvolveram um Manifesto para Melhoria de Processo de Software (SPI Manifesto) [Pries-Heje and Johansen 2010]. Este manifesto identifica três valores como os mais importantes: Pessoas, Negócio e Mudança. "Nós acreditamos firmemente que MPS (a) Deve envolver as pessoas ativamente e afetar as suas atividades diárias, não para exibição ou focalizada só na gerência; (b) É aquilo que você faz para o sucesso do negócio, não para cumprir uma norma, alcançar um nível de maturidade ou obter um certificado; e (c) É inerentemente ligada à mudança, não para continuar fazendo como fazemos hoje" Para cada um destes três valores foram identificados princípios. "Nós acreditamos que os princípios seguintes sustentam esses valores: (a) Pessoas: Conheça a cultura e foque nas necessidades, Motive todas as pessoas envolvidas, Baseie a melhoria na experiência e em medições, Crie uma organização que aprende; (b) Negócio: Dê sustentação à visão e aos objetivos de negócio, Use modelos dinâmicos e adaptáveis conforme a necessidade, e Aplique Gerência de Riscos; e (c) Mudanças: Gerencie as mudanças organizacionais no seu esforço de melhoria, Assegure que todas as partes entendam e concordem com o processo e Não perca o foco". Cada um dos três valores e dez princípios esta descrito no manifesto com o contexto, problema, explicações, sugestões e exemplos. Este manifesto foi traduzido para o português por uma equipe do CTI e publicado inicialmente como um Relatório Técnico com o objetivo de melhorar a disseminação dos valores e princípios da MPS no Brasil [Tsukumo et al. 2010]. O método PRO2PI-WORK está alinhado com os valores e princípios deste manifesto ao expandir as referências da melhoria para um conjunto mais abrangente.

\section{Objetivos e Metodologia}

O objetivo deste artigo é introduzir um método para o estabelecimento de referências que orientam um ciclo de melhoria de processo em uma organização intensiva de software ou outros trabalhos de conhecimento. Esta melhoria é integrada com os objetivos estratégicos da organização e é dirigida por um Perfil de Modelagem de Processo estabelecido para a organização como um perfil integrado com tipos de modelos de processo: Perfil de Capacidade de Processo, Descrição da Realização de Processo e Indicadores de Desempenho de Processo.

A base para a utilização integrada dos três tipos de modelos foi o conjunto de conceitos relacionados a modelo e modelagem, conforme descritos por Bézivin (2005) e outros, aplicados a processo e a um ciclo de melhoria de processo conforme descrito em outros artigos [Salviano et al. 2010] [Salviano 2011] em termos de modelo de especificação de um processo a ser construído ou modelo descritivo de um processo já existente. Um modelo de Perfil de Capacidade de Processo está estruturado por áreas de processos e níveis de capacidade, conforme definido, por exemplo, pelo CMMI [SEI 2010]. Um modelo de Descrição da Realização de Processo está estruturado por ciclos 
de vida, papéis, atividades e artefatos, definidos, por exemplo, pelo metamodelo SPEM (Software \& Systems Process Engineering Meta-Model) [OMG 2008]. Um modelo de Indicador de Desempenho de Processo está estruturado por necessidades de informação, produtos de informação, indicador e medidas, definido, por exemplo, pela Norma ISO/IEC 15939 (2008).

Este método está sendo desenvolvido utilizando como metodologia de pesquisa a abordagem "indústria-como-laboratório" (industry-as-laboratory) por meio de ciclos de demanda, exploração, aplicação e consolidação, proposto por Potts (1998). O desenvolvimento do método PRO2PI-WORK foi iniciado em 2005. Até 2009, todas as versões consideravam apenas Perfil de Capacidade de Processo como referência para a melhoria [Salviano 2009]. Foram realizadas cerca de trinta aplicações práticas com estas versões. Como todo método cada uso inclui uma customização do método. Nestas aplicações práticas foi percebido que dois tipos de customizações aconteciam com mais freqüência. Por isto foram definidas e utilizadas duas customizações do método: Uma com ênfase nas avaliações de processo em relação a um Perfil de Capacidade de Processo (PRO2PI-WORK4A - PRO2PI-WORK for Assessment) e outra com ênfase no ensino (educação) de melhoria de processo de software (PRO2PI-WORK4E PRO2PI-WORK for Education). No final de 2009 foi percebida a oportunidade de melhoria do método com a inclusão dos dois tipos de modelos de referência. Foi então desenvolvida uma nova versão da customização PRO2PI-WORK4A que foi utilizada em mais cinco aplicações [Salviano et al. 2010]. Este artigo apresenta uma nova versão do método PRO2PI-WORK na qual foi generalizada a inclusão dos novos dois tipos de referência do PRO2PI-WORK4A e foi incluída a possibilidade de estabelecimento de requisitos para os tipos de referência para os casos onde a empresa ainda não esteja preparada para o estabelecimento dos modelos. Esta nova versão foi utilizada em uma aplicação prática em 2011.

\section{Método PRO2PI-WORK}

PRO2PI-WORK, é um método para oficina (workshop) que tem como propósito estabelecer o PRO2PI (Perfil de Modelagem de Processo para melhoria de processo da organização). Este método foi desenvolvido para orientar uma melhoria de processo durante as fases de um ciclo de melhoria.

Este método utiliza a teoria de modelagem para orientar uma melhoria de processo em três referências de modelos: Perfis de Capacidade de Processo, Descrição da Realização de Processo e Indicadores de Desempenho de Processo. É um método inovador para estabelecimento destes três tipos de referências de modelos durante as fases de um ciclo de melhoria. O termo estabelecimento é utilizado em um sentido mais amplo incluindo a identificação e definição de modelos de processo para orientar a melhoria, o entendimento e revisão destes modelos pela organização e a avaliação dos processos em relação a estes modelos de referência.

Os objetivos definidos para este método são: a. Capacitar pessoas da organização em fundamentos e técnicas da melhoria de processo e elementos de modelos de referência; b. Consolidar informações relevantes sobre a organização; c. Estabelecer requisitos para um perfil de modelagem de processo; d. Estabelecer um perfil de modelagem de processo; e. Entender processos atuais em relação ao perfil 
definido; f. Definir orientações para atingir o perfil; e g. Reforçar motivação para melhoria de processo.

A versão atual do método PRO2PI-WORK tem quatro fases seqüenciais: Preparação (Fase 1), Análise de Referências (Fase 2), Consolidação de Referências (Fase 3) e Conclusão (Fase 4) conforme apresentado na Figura 1. As figuras 1 e 2 apresentam uma descrição gráfica, alto nível, do método PRO2PI-WORK e suas fases usando Business Process Modeling Notation - BPMN (2011). As fases 1 e 4 são executadas off-site e as fases 2 e 3 são executadas on-site na organização.
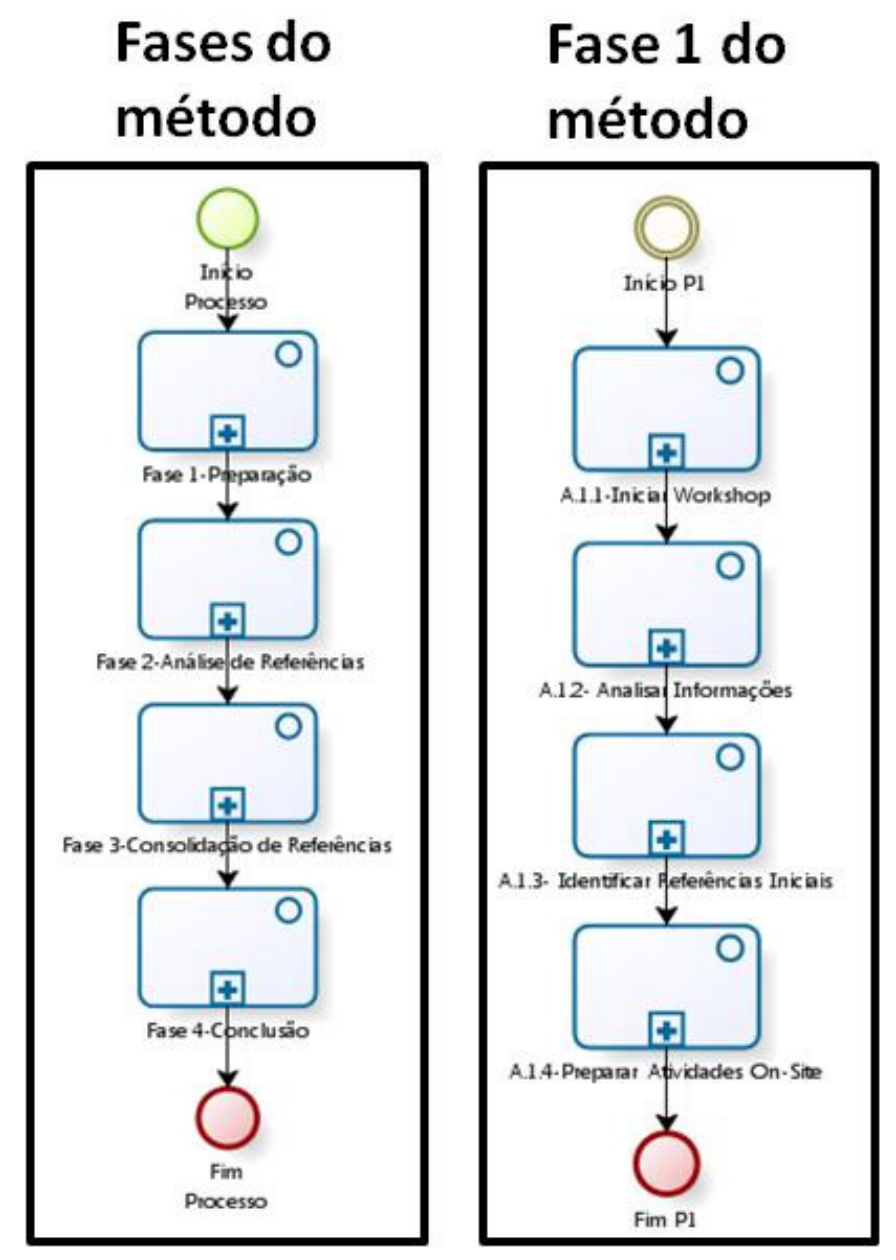

Figura 1. Fases do PRO2PI-WORK e atividades da Fase 1

A Fase 1 tem quatro atividades (Figura 1). Atividade A.1.1 Iniciar Workshop orienta a realização dos primeiros contatos com o patrocinador do workshop a fim de elicitar as necessidades e objetivos da organização-alvo deste workshop, auxiliando na customização do método para produzir o processo a ser executado. Durante esta atividade o planejamento é iniciado, com definição das datas para as atividades on-site, a equipe do workshop, as referências iniciais e outras questões.

A Atividade A.1.2 Analisar Informações orienta a obtenção e análise de informações relevantes sobre a organização, a fim de entender o contexto, objetivos e as necessidades de negócios da organização. É realizada a revisão do planejamento inicial com o patrocinador do workshop. Durante esta atividade é solicitado o preenchimento 
de um questionário para realizar este levantamento. A Atividade A.1.3 Identificar Referências Iniciais orienta a definição ou revisão das referências iniciais. Essas referências iniciais são compostas pelos requisitos e uma proposta inicial de três tipos de referências de modelo para o workshop. Dependendo das necessidades e objetivos, uma ou duas referências podem ser mais enfatizadas no workshop. A Atividade A.1.4 Preparar Atividades On-Site orienta o planejamento e preparação para as atividades on-site. O planejamento inclui a agenda, o cronograma, número de participantes do workshop e outras questões. Para a preparação, os especialistas do workshop elaboram os materiais relacionados com as referências iniciais.

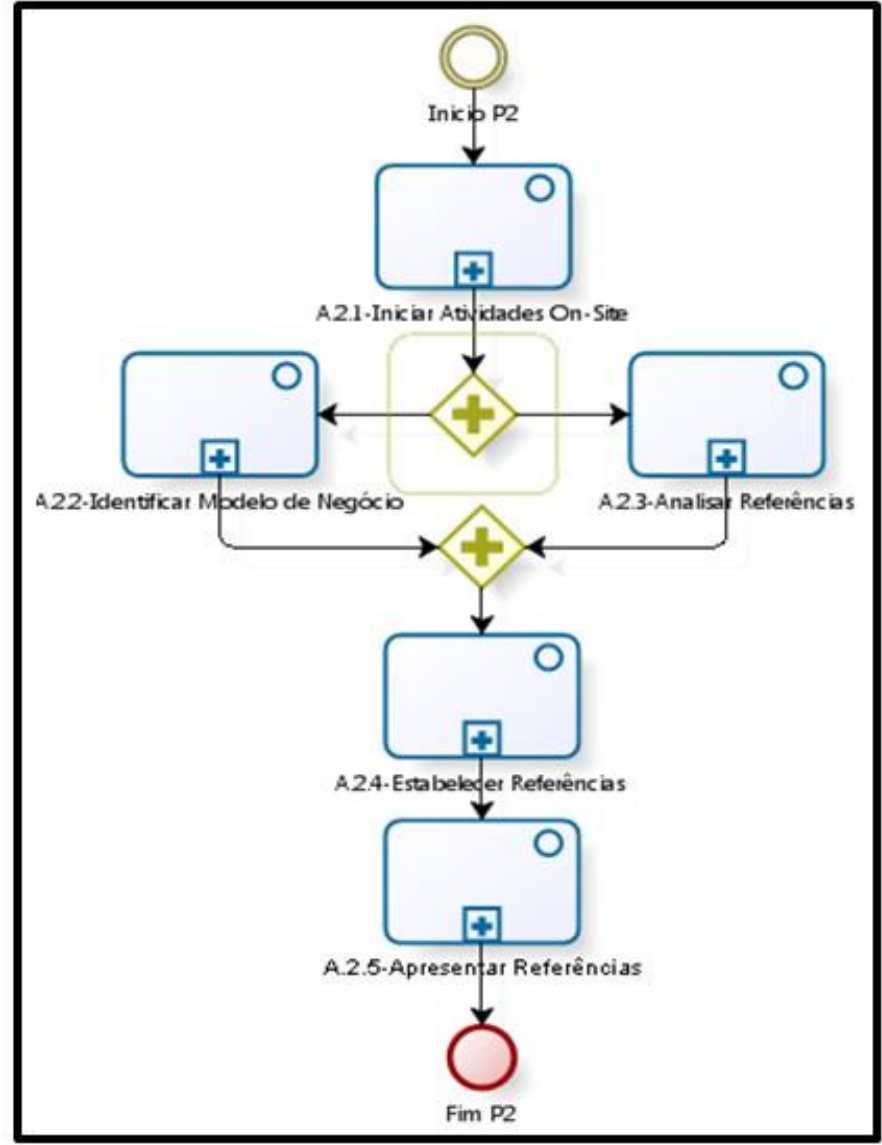

Fase 2

Fase 4

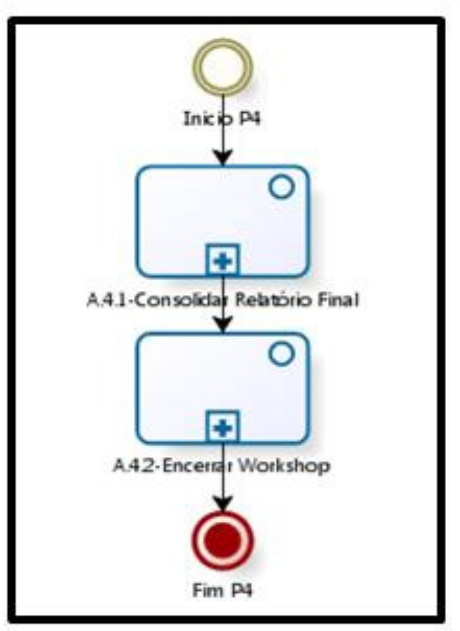

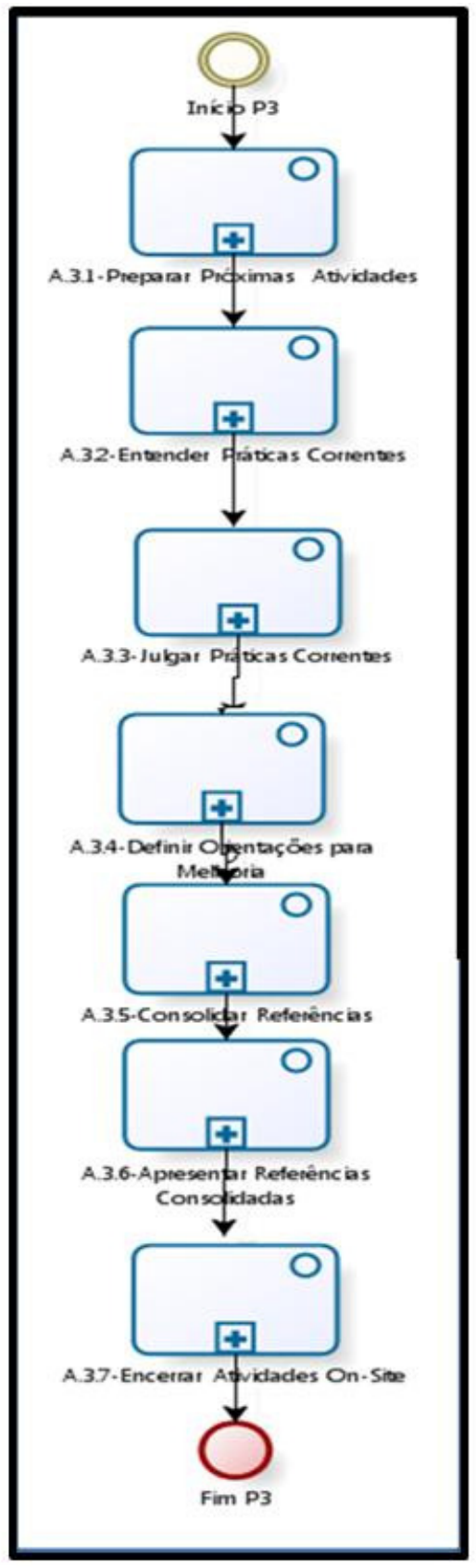

Fase 3

Figura 2. Fases 2, 3 e 4 do PRO2PI-WORK 
A Fase 2 tem cinco atividades (Figura 2). Atividade A.2.1 Iniciar Atividades On-Site orienta a apresentação inicial sobre os objetivos e características do workshop para as pessoas da organização que irão participar do workshop. A Atividade A.2.2 Identificar Modelo de Negócio orienta a identificação do modelo de negócio e a estratégia a fim de entender os pontos fortes, fraquezas, oportunidades e ameaças da organização. Essas informações irão auxiliar na customização do método para produzir o processo a ser executado. A Atividade A.2.3 Analisar Referências orienta a identificação e análise das referências relevantes para a organização. Para cada referência selecionada identificar três pontos: o quão bem elas são desempenhadas, a sua importância e o risco para a organização. A Atividade A.2.4 Estabelecer Referências orienta o estabelecimento das referências identificadas inicialmente, relevantes para a organização. Essas referências são compostas pelos requisitos e uma proposta inicial de três tipos de referências para o workshop: Perfis de Capacidade de Processo, Descrição da Realização de Processo e Indicadores de Desempenho de Processo. Dependendo das necessidades e objetivos, uma ou duas referências podem ser mais enfatizadas no workshop. A Atividade A.2.5 Apresentar Referências orienta a apresentação das referências ao patrocinador, podendo ser ajustadas essas referências.

A Fase 3 tem sete atividades (Figura 2). A Atividade A.3.1 Preparar Próximas Atividades orienta a preparação da equipe de workshop para a realização das próximas atividades, incluindo rever o material relacionado com as referências definidas, cronograma e outras questões. A Atividade A.3.2 Entender Práticas Correntes orienta a verificação dos dados dos projetos ou dados da organização a fim de obter um entendimento sobre as práticas correntes utilizando a análise nos documentos, entrevistas e outras técnicas. A Atividade A.3.3 Julgar Práticas Correntes orienta o julgamento dos dados utilizando o entendimento da equipe de workshop sobre as práticas correntes. Neste momento os modelos de especificação podem ser revisados para ajustes (referências originais).

A Atividade A.3.4 Definir Orientações para Melhoria orienta as definições de orientações para melhoria das situações das práticas correntes para uma especificação. Novamente neste momento os modelos de especificação podem ser revisados para ajustes (referências originais). A Atividade A.3.5 Consolidar Referências orienta a consolidação das referências obtidas utilizando o entendimento da equipe de workshop sobre as práticas correntes. Neste momento são preparados os resultados e os modelos de especificação e descrição podem ser revisados para ajustes. A Atividade A.3.6 Apresentar Referências Consolidadas orienta a apresentação das referências, geralmente com um relatório, uma apresentação e uma sessão executiva com o patrocinador. Também são apresentadas as orientações de melhorias. A Atividade A.3.7 Encerrar Atividades On-Site orienta a conclusão das atividades on-site.

A fase 4 tem duas atividades (Figura 2). A Atividade A.4.1 Consolidar Relatório Final orienta a consolidação do relatório final com o contexto e os resultados do workshop. Este relatório é encaminhado ao patrocinador para a sua aceitação. A Atividade A.4.2 Encerrar Workshop orienta a conclusão do workshop. Isso inclui uma análise sobre o workshop e possíveis orientações para a melhoria do método.

Cada uma destas 18 atividades estão descritas em uma estrutura baseada nos elementos da notação ETVX [Radice and Phillips 1988] composta pelos elementos 
Propósito, Atores, Produtos de Entrada, Produtos de Saída e Tarefas. Os parágrafos a seguir apresentam um resumo da descrição da Atividade A.2.3.

Propósito: Orientar a identificação ou revisão das referências relevantes para a organização. Para cada referência selecionada identificar três pontos: o quão bem elas são desempenhadas, a sua importância e o risco para a organização.

Atores: Especialista, Equipe de Workshop

\section{Produtos de Entrada:}

- Art03-ApresentacaoPRO2PI-WORK-Processos;

- Art05-RelevAreaCapProcUO [template]: Questionário sobre relevância de uma Área de Capacidade de Processo para uma Unidade Organizacional;

- Art06-QuadroRelevAreaProcUO [template]: Quadro resumido da relevância das áreas de processo analisadas para a unidade organizacional, em termos de importância e risco.

\section{Produtos de Saída:}

- Art05-RelevAreaCapProcUO [preenchido para cada processo]: Questionário sobre relevância de uma Área de Processo para uma Unidade Organizacional;

- Art06-QuadroRelevAreaProcUO [preenchido com todos os processos]: Quadro resumido da relevância das áreas de processo analisadas para a unidade organizacional, em termos de importância e risco.

\section{Tarefas:}

- Para cada processo definido pelas referências de Modelo de Perfil de Capacidade de Processo, as seguintes tarefas são realizadas, com uma estimativa de 40 minutos por processo:

- Especialista apresenta o processo para a equipe do workshop, a fim de obter um entendimento sobre as práticas correntes para avaliar as referências, por: a) definição do processo, conforme o modelo; b) sintomas típicos quando não é bem executado, e c) benefícios típicos porque é importante;

- Discutir e identificar a correspondência dele na organização;

- Identificar como ele é executado hoje, incluindo uma estimativa do seu nível de capacidade para a referência Perfil de Capacidade de Processo;

- Definir a importância desta área de processo para os objetivos de negócios da organização, expressada numa escala de três valores: baixa, média ou alta;

- Definir o risco desta área de processo, em relação ao nível de capacidade atual, observando os problemas típicos de sua não execução, se a área continuar a ser executada como é atualmente, expressada numa escala de três valores: baixa, média ou alta;

- Posicioná-lo no quadro em função das respostas de importância e risco e rever estas respostas.

- Para cada referência dos outros dois tipos de modelo são realizadas tarefas semelhantes, também com uma estimativa de 40 minutos por processo. 


\section{Considerações sobre uma utilização do método}

Conforme descrito anteriormente, o método pode ser utilizado em qualquer fase de um ciclo de melhoria. Esta seção descreve considerações sobre esta utilização, relata uma utilização prática do método e faz uma análise dos resultados.

\subsection{Considerações sobre a Utilização do Método}

Usando como exemplo um ciclo de melhoria de processo na visão de modelagem de processo, no inicio de um ciclo de melhoria temos que levantar as referências iniciais, isto é, em relação à referência Perfil de Capacidade de Processo quais as melhores práticas que podem ajudar a organização a alcançar os objetivos de negócios, como exemplo, buscar o nível F do MPS.BR, representando o modelo de especificação do Perfil de Capacidade de Processo para o processo futuro. Em relação à Referência do tipo Indicador de Desempenho de Processo qual o objetivo de medição que queremos atingir, como exemplo, reduzir a margem de erro nos projetos, que representa o Modelo de Especificação para Indicador de Desempenho de Processo para o processo futuro. Em relação à Referência do tipo Descrição da Realização de Processo quais os requisitos e características de uma descrição de processo adequada, como exemplo, num ciclo de vida incremental iterativo, que representa o Modelo de Especificação da Descrição da Realização de Processo.

$\mathrm{Na}$ atividade avaliar as práticas correntes, temos que analisar as referências iniciais, estabelecer as referências, entender, julgar e consolidar as práticas correntes, definir orientações de melhorias e apresentar estas referências aos responsáveis. Para isso, no Perfil de Capacidade de Processo é realizada uma avaliação nos processos, como exemplo, estimar o nível de capacidade dos processos em relação ao modelo MPS.BR, que representa o Modelo Descritivo do Perfil de Capacidade de Processo do processo atual; em relação a Referência Descrição da Realização de Processos, avaliar se os processos são curtos e usáveis para o ciclo de vida incremental iterativo, que representa o Modelo Descritivo da Descrição da Realização de Processos do processo atual, e em relação a Referência Indicadores de Desempenho de Processo avaliar se os projetos estão tendo muito problemas de atraso, custo, e outros pontos, que representa o Modelo Descritivo do Indicadores de Desempenho de Processo do processo atual.

$\mathrm{Na}$ atividade institucionalizar as melhorias a organização quer saber se estão usando as melhores práticas do nível $\mathrm{F}$ do MPS.BR e novamente acontece um novo ciclo de melhoria para orientar as ações e avaliar os resultados nos três tipos de modelos de referências. A utilização destes três tipos de modelos de referência de forma integrada permite uma melhor orientação da melhoria para atingir os objetivos de melhores resultados de negócio, satisfação das pessoas e gestão das mudanças. Esta é a proposta do PRO2PI-WORK.

\subsection{Relato de uma Utilização Prática do Método}

A versão atual do método descrito neste artigo foi utilizada em fevereiro de 2011, numa empresa especializada em sistemas eletroeletrônicos de veículos, e essa experiência será apresentada nesta seção, descrita em termos de atividades do método.

Durante a Atividade A.1.1 Iniciar Workshop houve os primeiros contatos com os patrocinadores do workshop, com o esclarecimento das necessidades e objetivos da empresa contratante, a customização do método para esta utilização, a determinação da 
data da realização das atividades on-site, definição da equipe e dos participantes do workshop, e outras atividades para iniciar o workshop. As atividades on-site foram definidas para de 22 a 24 de fevereiro de 2011 com uma equipe composta por dois pesquisadores do CTI e quatro representantes da empresa. As principais necessidades da empresa foram relacionadas a treinamento no modelo Automotive SPICE, uma avaliação em relação a um conjunto de processos do Automotive SPICE e orientações para melhoria de processo.

Dado que existe uma demanda por padrões mais apropriados para a indústria automobilística, grandes fabricantes de automóveis europeus iniciaram em 2001 o desenvolvimento do Automotive SPICE [Automotive SIG 2010] como um modelo para avaliação de processo compatível com a Norma ISO/IEC 15504 (SPICE). A referência principal foi o modelo ISO/IEC 15504-5. Este modelo define 48 processos e seis níveis de capacidade. Para o Automotive SPICE foram selecionados e adaptados alguns processos da ISO/IEC 15504-5 e foram também definidos novos processos. O desenvolvimento do Automotive SPICE foi realizado pelo Automotive Special Interest Group (SIG) junto com o The Procurement Forum e o SPICE User Group. Os fabricantes de automóveis alemães Audi, BMW Group, Daimler AG, Porsche e Volkswagen estão trabalhando juntos no HIS (Herstellerinitative Software, Hersteller Initiative Software) para padrões conjuntos nas áreas de software, unidades de controle eletrônicas, avaliação de processo, simulação e ferramentas. O HIS Scope foi definido para orientar a melhoria e avaliação de processos com o Automotive SPICE. Várias montadoras utilizam este perfil para avaliar seus fornecedores. A empresa definiu como o Perfil de Capacidade de Processo para este workshop os 15 processos do HIS Scope mais o processo de Medição, todos no nível 2 de capacidade. Estes relacionamentos entre o modelo ISO/IEC 15504-5, o modelo Automotive SPICE e os 16 processos do HIS Scope mais o processo de medição estão ilustrados na Figura 3.

\section{De ISO/IEC 15504-5 para Automotive SPICE para HIS Scope}

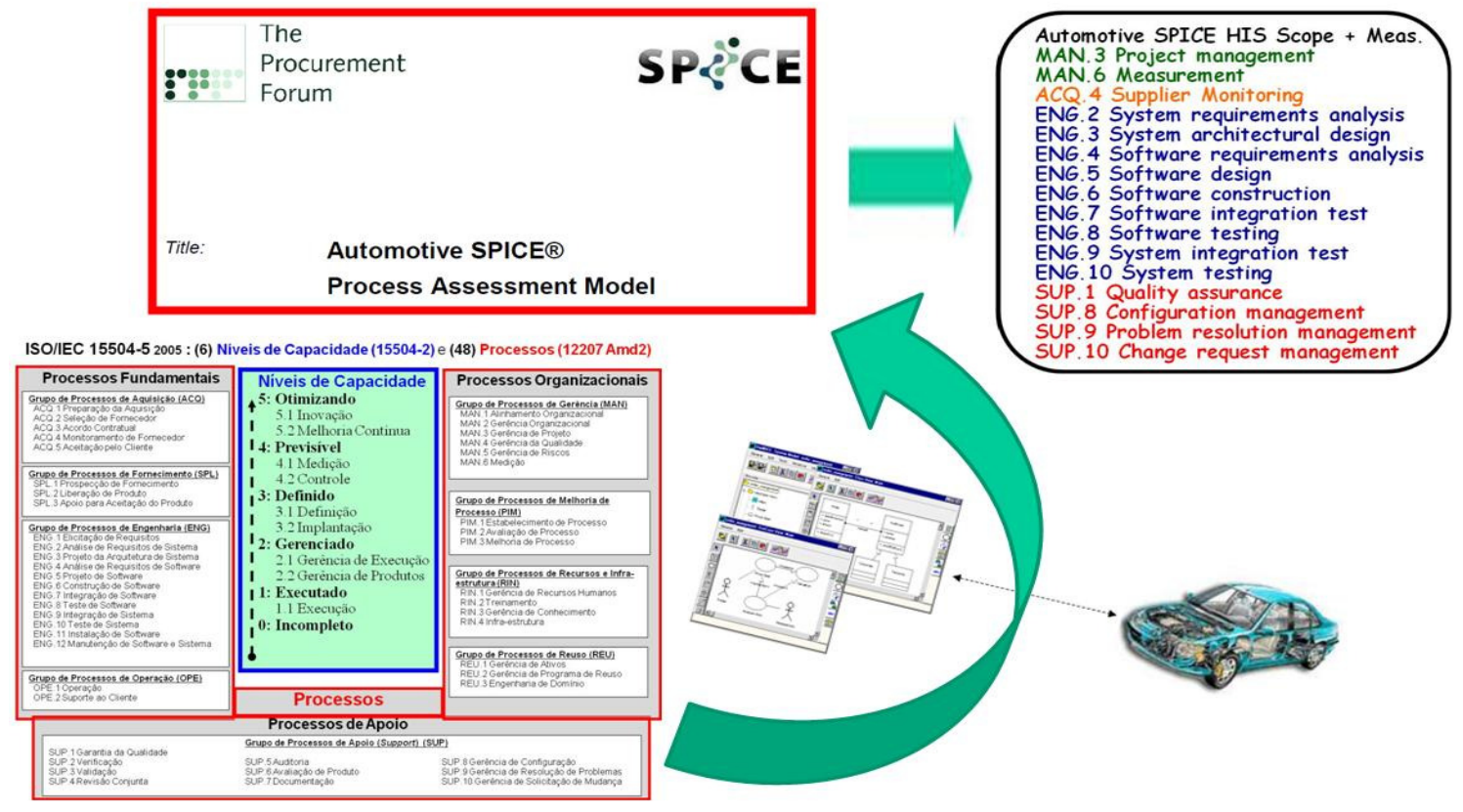

Figura 3. ISO/IEC 15504-5, Automotive SPICE e HIS Scope 
Com a experiência do CTI e as orientações do método PRO2PI-WORK, estas necessidades foram transformadas e acordadas com o cliente nos objetivos do workshop. Estes objetivos foram: capacitar pessoas da empresa em fundamentos e técnicas da melhoria de processo e elementos do modelo Automotive SPICE, consolidar informações relevantes sobre a empresa; estabelecer requisitos para a Descrição da realização de Processos e para Indicadores de Desempenho de Processo, estabelecer um Perfil de Capacidade de Processo, entender processos atuais em relação ao Perfil de Capacidade de Processo, definir orientações para atingir o perfil, e reforçar a motivação para melhoria de processo.

O método então foi customizado produzindo o processo a ser utilizado. Esta customização utilizou elementos das duas customizações já pré-definidas, pois os objetivos incluíam o treinamento em melhoria de processo e no modelo e o foco principal de avaliação em relação a um Perfil de Capacidade de Processo. O treinamento em Melhoria de Processo de Software foi baseado no SPI Manifesto. Como o Perfil de Capacidade de Processo já foi escolhido, o objetivo passou a ser mais a confirmação ou não da adequação deste perfil. O estabelecimento dos outros dois tipos de perfis foi substituído pelo estabelecimento de requisitos para estes perfis. Com isto optou-se em juntar as atividades A.2.3 Analisar Referências, A.3.2 Entender Práticas Correntes e A.3.3 Julgar Práticas Correntes em uma única atividade.

Durante a Atividade A.1.2 Analisar Informações foi obtida a informação de que a empresa realizou ciclos de melhorias nos seus processos de desenvolvimento de firmware com o modelo CMMI-DEV e foi avaliada no nível 2 de maturidade em 2008. Em 2009 foi realizada uma auditoria externa utilizando o modelo Automotive SPICE, avaliando cada processo que faz parte do HIS Scope no nível 1 de capacidade. Mais informações foram repassadas pela empresa a fim de entender o contexto, objetivos e as necessidades de negócios.

Durante a Atividade A.1.3 Identificar Referências Iniciais foram revisadas as referências iniciais e acordado que o Perfil de Capacidade de Processo para esta oficina é composto pelos 15 processos do HIS Scope mais o processo MAN.6 - Measurement, cada um no nível 2 de capacidade. Para a referência Indicadores de Desempenho de Processo foi considerado como sendo o modelo lógico definido na Norma ISO/IEC 15939 (2008). Para a referência Descrição da Realização de Processo foi considerado um conjunto de requisitos para orientação de uma boa descrição de processo tendo como a principal referência uma proposta de Olson (2006). Durante a Atividade A.1.4 Preparar Atividades On-Site foram elaboradas a agenda e as apresentações relacionadas com as referências identificadas. Como este foi o primeiro workshop que utilizou o modelo Automotive SPICE foram desenvolvidos slides sobre os processos com base nos slides já desenvolvidos para o modelo ISO/IEC 15504-5.

Durante a Atividade A.2.1 Iniciar Atividades On-Site foi apresentado e discutido o SPI Manifesto [Pries-Heje and Johansen 2010]. Também foram apresentados e discutidos uma visão geral do modelo Automotive SPICE e fundamentos do método PRO2PI-WORK. Durante a Atividade A.2.2 Identificar Modelo de Negócio o patrocinador do workshop apresentou a empresa (missão, visão, objetivos de negócio, organograma, entre outros) e foi identificado que a empresa desenvolve produtos eletroeletrônicos e mecatrônicos de veículos e motocicletas para as indústrias automobilísticas. O modelo de negócio da empresa é diferente dos modelos mais 
tradicionais que variam entre desenvolver produtos e desenvolver projetos. A empresa geralmente estabelece um contrato com uma montadora para desenvolver um sistema e produzir uma determinada quantidade deste sistema com uma linha de montagem. $\mathrm{O}$ escopo da melhoria de processo é o projeto do sistema.

Durante a Atividade A.2.3 Analisar Referências foram apresentados e discutidos detalhadamente os 16 processos que fazem parte do HIS Scope e outros elementos do modelo Automotive SPICE a fim de obter um entendimento e uma avaliação sobre as práticas correntes e também identificando a sua relevância para as referências [Figura 4]. Foram discutidos e analisados três pontos: o quão bem elas são executadas para estimar o nível de capacidade de cada processo, a sua importância para a empresa (expressa em termos de importância baixa, média ou alta) e o risco para a empresa se este processo continuar sendo realizado da forma que é atualmente (expresso em termo de baixo, médio ou alto). $\mathrm{O}$ resultado foi resumido em uma matriz na qual cada processo foi posicionado em termos de importância e risco.

Durante a Atividade A.2.4 Estabelecer Referências foi confirmado que os processos que fazem parte do HIS Scope são apropriados para os modelos de referências estabelecidos. Durante a Atividade A.2.5 Apresentar Referências foi apresentada e discutida com a equipe de workshop uma versão preliminar dos resultados da avaliação dos processos em relação aos modelos de referências. As estimativas do nível de capacidade de cada processo do HIS Scope Automotive SPICE e o quadro de relevância das áreas de processo analisadas em termos de importância e risco foram discutidas.

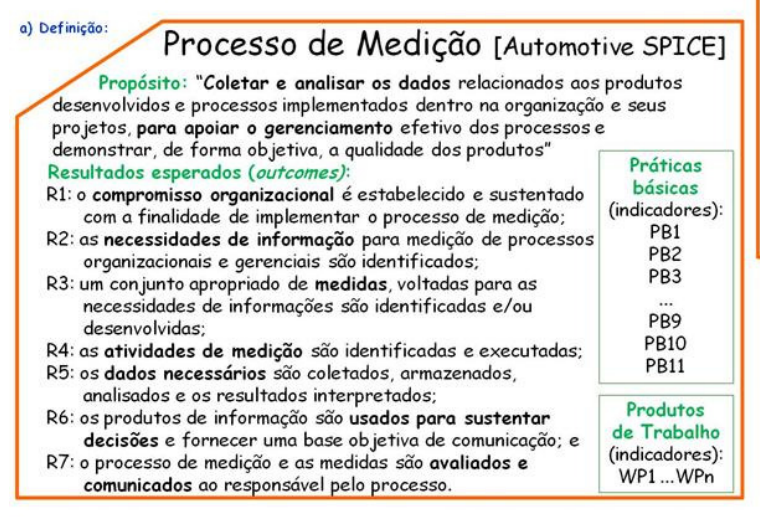

Figura 4. Orientações para Analisar Referência relativa ao processo de Medição

Durante a Atividade A.3.4 Definir Orientações para a Melhoria foram definidos dois perfis de ciclos de melhoria, sendo que o primeiro ciclo tem duas 
alternativas para sua implementação e o segundo ciclo complementa o primeiro ciclo, consolidando o Perfil de Capacidade de Processo. As duas alternativas do primeiro ciclo de melhoria definidas foram: 1) expansão do escopo da melhoria de processo para a área de Sistemas, e 2) escopo de melhoria de processo nas áreas de engenharia de software. Durante a Atividade A.3.5 Consolidar Referências foram revisados e consolidados os 02 perfis de ciclos de melhoria em relação do modelo de referência Perfil de Capacidade de Processo. Também foram confirmadas as referências relacionadas ao Perfil de Indicadores de Desempenho de Processo e Descrição da Realização de Processo.

Durante a Atividade A.3.6 Apresentar Referências Consolidadas foram apresentados e discutidos com os patrocinadores do workshop os resultados da avaliação dos processos em relação aos modelos de referências. As estimativas do nível de capacidade de cada processo do HIS Scope Automotive SPICE e o quadro de relevância das áreas de processo analisadas em termos de importância e risco foram apresentadas e discutidas. Também foram apresentados e discutidos duas alternativas para a melhoria que foram representadas como dois Perfis de Capacidade de Processo. A empresa irá decidir o perfil a ser utilizado considerando as outras visões que podem interferir na definição da implementação dessas melhorias, como as necessidades e requisitos que devem ser atendidos vindos das montadoras, da matriz e da própria empresa. Por meio da Atividade A.3.7 Encerrar Atividades On-Site foram concluídas as atividades on-site.

Durante a Atividade A.4.1 Consolidar Relatório Final foi consolidado o relatório final como um Relatório Técnico do CTI (identificado TR0015111) e encaminhado aos patrocinadores. Por meio da Atividade A.4.2 Encerrar Workshop foi encerrado o workshop com uma análise dos seus resultados.

\subsection{Análise dos Resultados}

Os resultados foram analisados em termos dos objetivos deste workshop. Um dos representantes da empresa comentou no final do workshop que a mensagem do SPI Manifesto sobre os valores da MPS foi importante para entender a MPS. Outros comentários sinalizaram a capacitação de pessoas da empresa em fundamentos e técnicas da melhoria de processo e em elementos do modelo Automotive SPICE. Houve um entendimento das características da empresa. O Perfil de Capacidade de Processo resultante mostrou o estado atual dos processos em relação ao HIS Scope. Os requisitos para a Descrição da Realização de Processos e para Indicadores de Desempenho de Processo ajudaram a empresa a entender melhor as referências para a melhoria. Orientações para a melhoria foram definidas duas alternativas para a melhoria que foram representadas como dois Perfis de Capacidade de Processo.

Foi percebida a necessidade de maior disseminação da utilização dos dois tipos adicionais de referências para a melhoria: Descrição da Realização de Processos e para Indicadores de Desempenho de Processo.

A empresa fez uma análise comparativa em relação ao resultado da avaliação apresentado no workshop com o resultado da auditoria externa HIS Scope nível de capacidade 1 realizada em 2009 e reportou que um resultado complementou o outro, confirmando a viabilidade do uso do método PRO2PI-WORK nas organizações intensivas de software, mostrando significativo para facilitar a melhoria e avaliação de processos de software. 
Como próximos passos, o método está sendo descrito em mais detalhes para possibilitar sua utilização por outras pessoas além dos autores. A descrição do método SCAMPI está sendo utilizada como uma das referências para determinar o nível de detalhamento da descrição.

A inclusão nesta nova versão da possibilidade de trabalhar com os requisitos para as referências mostrou-se apropriada para os casos como o desta aplicação onde ainda não existe um entendimento dos três tipos de referências.

\section{Conclusão}

A principal inovação das versões mais recentes do método para estabelecimento de referências em ciclos de melhoria de processo é a utilização integrada dos três tipos de referências: Perfil de Capacidade de Processo, Descrição da Realização de Processos e Indicadores de Desempenho de Processo.

Análises das utilizações do método incluindo da utilização mais recente descrita neste artigo têm sinalizado o atendimento dos objetivos do método, com limitações que demandam melhorias. Uma destas limitações é alguma dificuldade no entendimento da utilização dos dois tipos adicionais de referências para a melhoria: Descrição da Realização de Processos e para Indicadores de Desempenho de Processo.

\section{Agradecimentos}

Os autores agradecem os revisores anônimos deste artigo no SBQS pelos seus comentários e sugestões que contribuíram para o aperfeiçoamento deste artigo.

\section{Referências}

[Automotive SIG 2010] Automotive SIG, Automotive SPICE® - Process Assessment Model, Technical Report, www.automotivespice.com, May 10th, 2010.

[Bézivin 2005] Bézivin, J. "On The Unification Power of Models", Software and System Modeling, 2005.

[BPMN 2011] Business Process Modeling Notation. BPMN 2.0 Especification. Disponível em: <http://www.bpmn.org/> Acesso em: 18/04/2011.

[HIS 2011] Herstellerinitative Software, Hersteller Initiative Software, Iniciativa Hersteller de Software, http://www.automotive-his.de/, ultimo acesso em 22/02/2011.

[ISO/IEC 2008] International Organization for Standardization and the International Electrotechnical Commission, ISO/IEC 15939 - Software engineering - Software measurement process, 37 pages, 2008.

[ISO/IEC 2004] ISO/IEC, ISO/IEC 15504, composed of seven parts (15504-1 to 155047) parts, under the general title Information technology — Process assessment, 20042008.

[McFeeley 1996] McFeeley, B., IDEAL - "A User's Guide for Software process Improvement”, Handbook CMU/SEI-96-HB-001, 236 pages.

[Olson 2006] Olson, Timothy G., "Defining Short and Usable Process". The Journal of Defense Software Engineering, San Diego, p. 24-28. 
[OMG 2008] Object Management Group, Software \& Systems Process Engineering Meta-Model Specification (SPEM), version 2.0, OMG Document Number: formal/2008-04-01, 218 pages, April 2008.

[Potts 1998] Potts, C. "Software-Engineering Research Revised", IEEE Software, 10(5), pp. 19-28.

[Pries-Heje and Johansen 2010] Pries-Heje, J., Johansen, J. (Chief Editors) (2010) "SPI Manifesto", eurospi.net, version A.1.2.2010.

[Radice and Phillips 1988] Radice, Ronald A. and Phillips, Richard W. Software Engineering, An Industrial Approach. Englewood Cliffs, New Jersey: Prentice Hall, 1988.

[Salviano 2009] Salviano, C. F., "A Multi-Model Process Improvement Methodology Driven by Capability Profiles”, Proc. of IEEE COMPSAC, Seatle, USA, p.636-637 (DOI 10.1109/COMPSAC.2009.94)

[Salviano 2011] Salviano, C. F., "A Modeling View of Process Improvement", Accepted to be published in: Proc. of SPICE International Conference, Dublin City University, Ireland, R.V. O'Connor et al. (Eds.), CCIS 155, pp. 16-27, (C) SpringerVerlag Berlin Heidelberg 2011.

[Salviano et al. 2010] Salviano, C.F., Martinez, M.R.M., Banhesse, E.L., Enelize, A., Zoucas, A., Thiry, M.: A, Method for Tridimensional Process Assessment Using Modelling Theory. In: Proc. of IEEE Seventh QUATIC, Porto, Portugal, pp. 430435 (2010), doi:10.1109/QUATIC.2010.95

[SEI 2010] SOFTWARE ENGINEERING INSTITUTE. CMMI for Development (CMMI-DEV), Version 1.3. Technical Report CMU/SEI-2010-TR-033, 2010.

[SEI 2011] SCAMPI Upgrade Team, Standard CMMI Appraisal Method for Process Improvement (SCAMPI) A, Version 1.3: Method Definition Document, HANDBOOK/ CMU/SEI-2011-HB-001, 2011.

[SOFTEX, 2009] Associação para Promoção da Excelência do Software Brasileiro. MR-MPS - Melhoria de Processo do Software Brasileiro - Guia de Avaliação. SOFTEX, Campinas, SP, 2009.

[Tsukumo et al. 2010] Tsukumo, A. N., Sampaio, A. L., Salviano, C. F., Primo, F. F., De Martino, W. R., Manifesto MPS, Tradução para o Português do SPI Manifesto, Relatório Técnico CTI DMPQS TRT0229110, 2010. 